\title{
Prevalence and Patterns of Comorbidity Among Middle-Aged and Elderly People in China: A Cross-Sectional Study Based on CHARLS Data
}

\author{
Zhao-Ya Fan' \\ Yuan Yang ${ }^{2}$ \\ Chang-Hong Zhang (iD) \\ Ruo-Yun Yin' \\ Lei Tang' \\ Fan Zhang' \\ 'School of Public Health and \\ Management, Research Center for \\ Medicine and Social Development, \\ Collaborative Innovation Center of Social \\ Risks Governance in Health, Chongqing \\ Medical University, Chongqing, 400016, \\ People's Republic of China; ${ }^{2}$ Department \\ of Cardiovascular Medicine, The First \\ Affiliated Hospital of Chongqing Medical \\ University, Chongqing, 400016, People's \\ Republic of China
}

Correspondence: Fan Zhang

School of Public Health and Management, Chongqing Medical University, No. I

Yixueyuan Road, Yuzhong District,

Chongqing, 4000I6, People's Republic of China

Tel +8613983782377

$\mathrm{Fax}+86 \quad 1368485068$

Email epicqmu@।63.com
Introduction: Under the background of the accelerated aging of the population, comorbidity in the elderly has gradually become a social problem. At present, the related studies on chronic diseases are mainly focused on a single disease. This study aimed to investigate the prevalence of common chronic diseases, the conditions and patterns of comorbidity in middle-aged and elderly people in China.

Methods: We extracted the data from China Health and Retirement Longitudinal Study (CHARLS). A total of 14 diseases were included, and the prevalence was assessed by selfreport. We calculate different disease combinations and perform descriptive statistics analysis of chronic disease and comorbidity status.

Results: Among the 6754 subjects, 2833 (42.0\%) people had at least one chronic disease, and $1138(17.0 \%)$ people had two or more diseases at the same time. The top three diseases of prevalence were hypertension $(15.4 \%)$, arthritis or rheumatism $(11.0 \%)$, and stomach or digestive diseases $(9.3 \%)$. Common dual disease combinations were hypertension and dyslipidemia, hypertension and arthritis or rheumatism, arthritis or rheumatism and stomach or digestive diseases.

Conclusion: Comorbidity is common in the population, and the pattern of chronic disease comorbidity is complex. Hypertension exists in a variety of comorbidity patterns, and its screening and prevention should be strengthened.

Keywords: chronic diseases, comorbidity, CHARLS, China

\section{Introduction}

Since China entered an aging society in 2000, the degree of population aging has continued to deepen. ${ }^{1}$ By the end of 2019 , the number of people aged 65 and above was 176 million, accounting for $12.6 \%$ of the total population, and is expected to reach 255 million in 2020 , accounting for $17.8 \%$ of the total population. ${ }^{2}$ Against that background, the prevalence of chronic non-communicable diseases in the middle-aged and elderly has increased rapidly, and often suffer from multiple diseases at the same time. In 2008, the World Health Organization (WHO) officially defined "suffering from two or more chronic diseases at the same time" as comorbidity. ${ }^{3}$ Comorbidity leads to the difficulty of treatment, the increase of drug use $\mathrm{e}^{4}$ and the aggravation of disease burden, which seriously affects the quality of life of patients. ${ }^{5,6}$ Since the outbreak of COVID-19 in early 2020, chronic comorbidity patients belong to the susceptible population, especially the elderly patients with poor prognosis and increased risk of death. Multimorbidity is a huge burden on individuals, households and countries. And the comorbidity of the middle-aged and the elderly has gradually become a public health problem affecting the 
sustainable development of the country. ${ }^{7}$ Studies on comorbidity of chronic diseases involve many fields, such as medicine, epidemiology, social economics, etc. Taking into account the different socio-demographic structure and disease patterns, the prevalence and patterns of comorbidity among the middleaged and elderly people in China may be different from those of other countries. ${ }^{8}$ At present, the domestic attention to the problem of comorbidity is still in its infancy, and the investigation is mostly conducted in local areas with a limited sample size. ${ }^{9}$ Previous studies have usually examined the differences between people with and without disease, but few have analyzed the different patterns of disease compositions associated with comorbidities. ${ }^{10}$ Therefore, our study intended to use the nationally representative data released by the China Health and Retirement Longitudinal Study (CHARLS) to analyze the status of comorbidity among middle-aged and elderly people in China, in order to provide theoretical basis and experience for comorbidity management.

\section{Materials and Methods Sample and Data}

We used cross-sectional data from the CHARLS Wave 4 (2018). CHARLS was an ongoing nationally representative cohort study involving a longitudinal survey of people aged 45 and over and their partners, started in 2011, followed up with participants every two years, and covered 450 villages in 150 counties of 28 provinces in China. Multistage, probability proportionate scale was used to collect the individual, family and additional social conditions of middle-aged and elderly people aged 45 and above in China. Data were collected by face-to-face computerassisted personal interviews with good response rate and credibility. Informed consent was obtained from all participants. The biomedical ethics committee of Beijing University approved the study, and detailed descriptions of the survey design and procedures were reported in the original study documentation. Details of CHARLS can be accessed elsewhere. ${ }^{11}$

According to the division of age limit by WHO, young people under 45 years old, middle-aged and elderly people aged 45 and above. The inclusion criteria for this study were as follows: (1) aged $\geq 45$ years; (2) complete information on age, gender, and prevalence of chronic disease. A total of 19,816 samples was collected in Wave 4, and the final number of the people included in this study after screening was 6754 .

\section{Measures and Analysis}

Chronic Disease and Comorbidities

In the CHARLS 2018 questionnaire, we extracted the data of "demographic backgrounds" and "health status and functioning". Among them, prevalence of chronic diseases was evaluated by asking "have you been diagnosed with [conditions listed below] by a doctor?", with diseases including hypertension; dyslipidemia (elevation of low-density lipoprotein, triglycerides, and total cholesterol, or a low high-density lipoprotein level); diabetes or high blood sugar; cancer or malignant tumor (excluding minor skin cancers); chronic lung diseases (such as chronic bronchitis or emphysema, excluding tumors or cancers); liver disease (except fatty liver, tumors, and cancer); heart attack (including coronary heart disease, angina, congestive heart failure, or other heart problems); stroke; kidney disease (except for tumor or cancer); stomach or other digestive diseases (except for tumor or cancer); emotional, nervous, or psychiatric problems; memoryrelated disease (such as dementia, brain atrophy, and Parkinson's disease); arthritis or rheumatism and asthma. A total of 14 common chronic diseases.

\section{Analysis}

In the descriptive analyses, continuous variables were reported as mean \pm standard deviation (SD) and categorical variables as percentages. Prevalence rate $=$ the number of people suffering from the disease/the total number of people * $100 \%$. Stata version 15.0 (Stata Corporation LP, College Station, TX, USA) was used for data cleaning. Microsoft Office Excel 365 (Microsoft, Redmond, WA, USA) was used to draw figures, and all statistics analysis was performed with SPSS version 20.0 (SPSS, Inc., Chicago, IL, USA).

\section{Results}

\section{Sample Description}

A total of 6754 samples was included in this study. The oldest was 97 years old, 3314 (49.1\%) were male, with an average age of $58.10 \pm 9.85$ years, and 3440 (50.9\%) were female, with an average age of $59.55 \pm 9.78$ years. A total of 3921 (58.1\%) people did not suffer from the disease, 1695 (25.1\%) suffered from one disease, and 1138 (16.9\%) suffered from two or more diseases. Divided into five groups according to age, there were 3923 (58.1\%) people aged 45-59, with an average age of 51.87 \pm 3.73 years, $1850(27.4 \%)$ people aged $60-69$, with an average age of $64.14 \pm 2.75$ years, 697 (10.3\%) people aged 70-79, with an average age of $73.72 \pm 2.81$ years, 256 (3.8\%) people aged $80-89$, with an average age of $83.02 \pm 2.50$ years, 28 
A

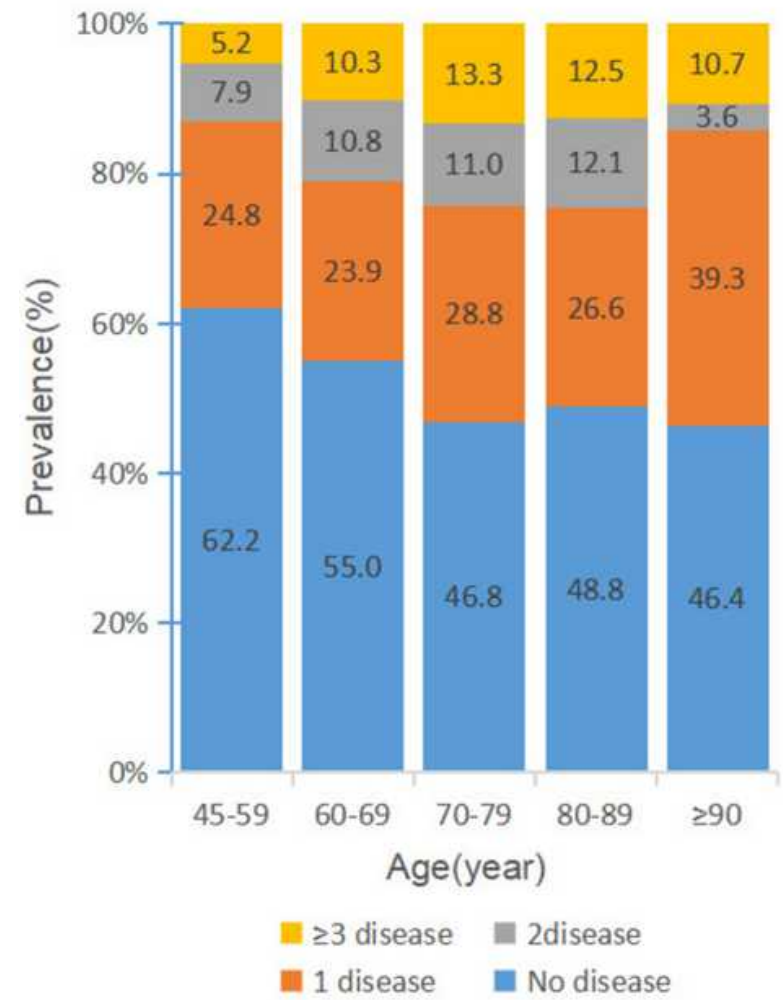

B

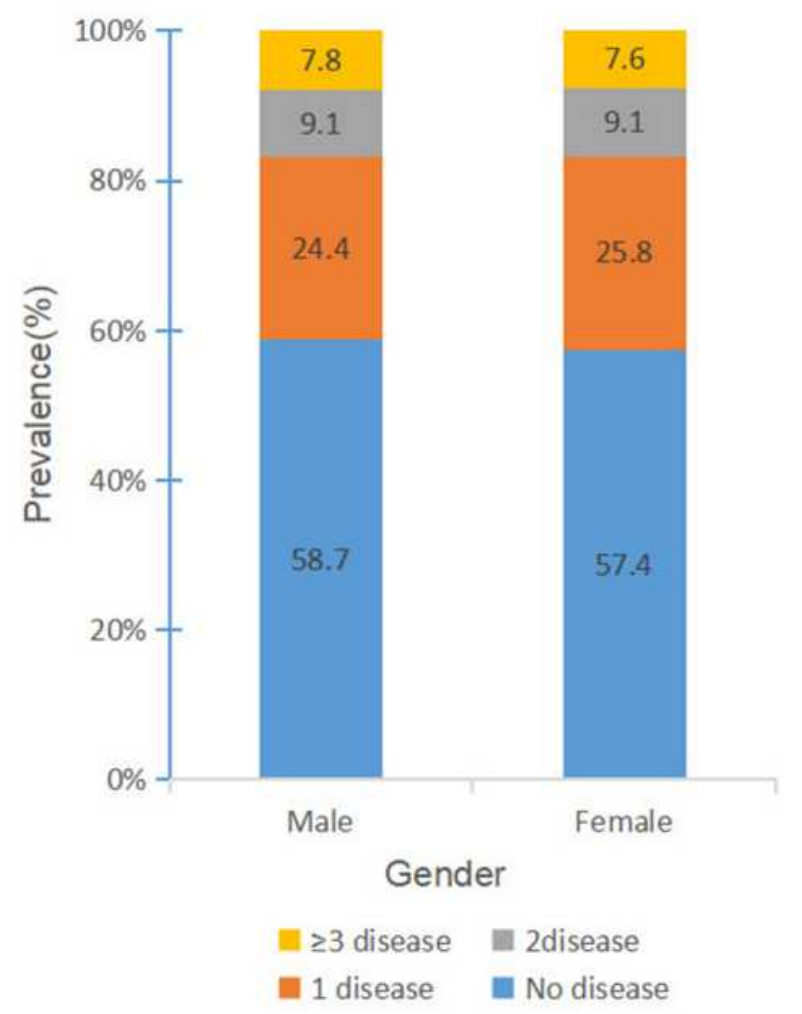

Figure I Chronic diseases prevalence by age (A) and gender (B) among middle-aged and elderly Chinese.

$(0.4 \%)$ people aged $\geq 90$, with an average age of $92.75 \pm 2.19$ years. The specific distribution is shown in Figure 1.

\section{Prevalence of Chronic Diseases}

Table 1, Table 2 summarizes the number of patients with different number of diseases. In terms of the number of chronic diseases, $2833(42.0 \%)$ patients suffered from at least one disease in the whole sample, and the maximum number of diseases was 11 , only one person. With the increase of the number of diseases, the total number of patients decreased significantly. Figure 2 shows the number of patients with various chronic

Table I Basic Information of the Number of Chronic Diseases

\begin{tabular}{|l|c|c|c|}
\hline No. of Diseases & $\mathbf{N}$ & No. of Diseases & $\mathbf{N}$ \\
\hline 0 & 3921 & 6 & 25 \\
$\mathrm{I}$ & 1695 & 7 & $\mathrm{II}$ \\
2 & 616 & 8 & 5 \\
3 & 275 & 9 & 2 \\
4 & 147 & $\mathrm{II}$ & $\mathrm{I}$ \\
5 & 56 & & \\
\hline
\end{tabular}

diseases in the survey. The top 3 prevalence rates of 14 chronic diseases were hypertension (15.4\%), arthritis or rheumatism $(11.0 \%)$, stomach or other digestive diseases $(9.3 \%)$.

\section{Prevalence of Chronic Comorbidities}

The results showed that the prevalence of comorbidity among the population was $16.8 \%$. Among the 1138 patients with comorbidities, there were 616 (54.1\%) patients have two diseases, with a total of 74 combinations, and $275(24.2 \%)$ patients have three diseases, with a total of 62 combinations. The top binary comorbidities and ternary comorbidities combinations are shown in Table 3 .

\section{Discussion}

The prevalence of comorbidity among the elderly varies greatly in different countries and regions, ${ }^{12}$ which may be related to the age of the population, the number of chronic diseases involved and the sampling framework. Our results showed that $42 \%$ of the middle-aged and elderly people had chronic diseases, and the prevalence of comorbidities 


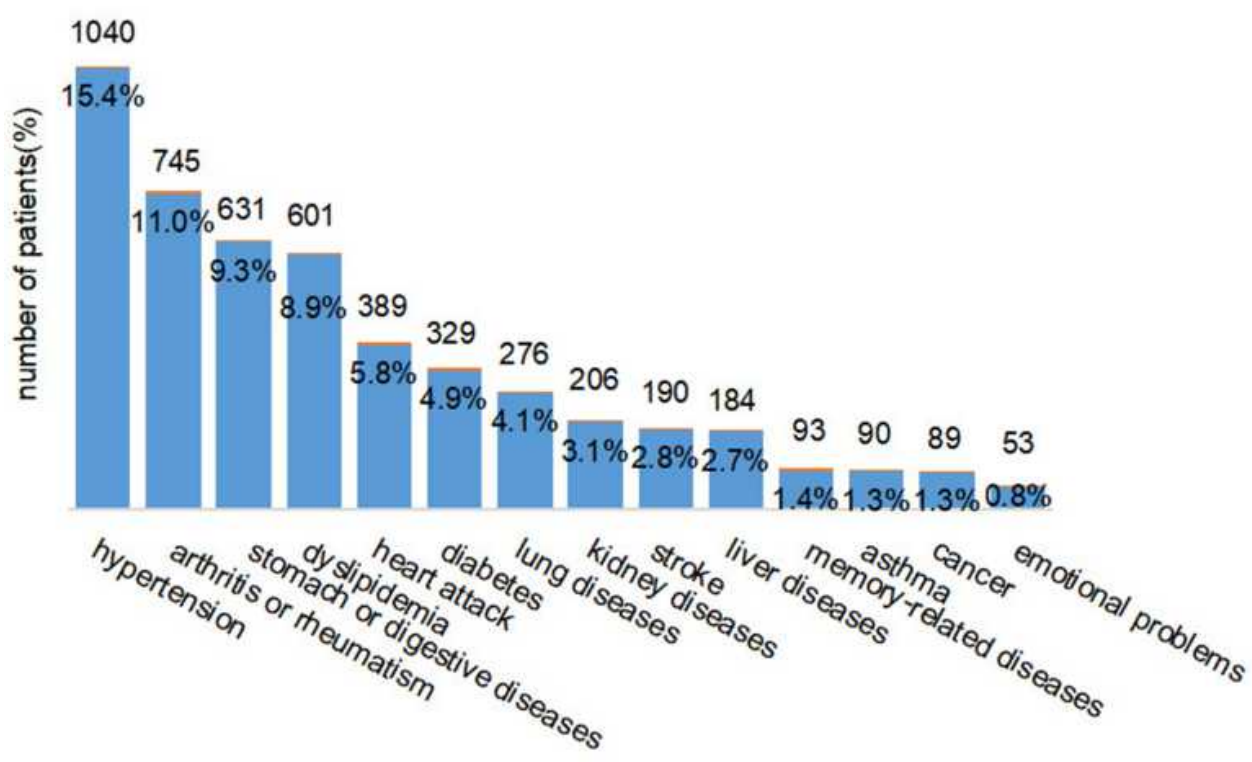

Types of chronic diseases

Figure 2 Distribution of the number of patients with the disease.

was $16.8 \%$. Similar to other studies, ${ }^{13-16}$ the combination of hypertension and dyslipidemia was the most frequent coupling among individuals with two conditions, the combination of hypertension, dyslipidemia and diabetes was the most frequent mix among those with three conditions. Epidemiological studies have shown that patients with hypertension have a high risk of developing diabetes.
The reason may be that insulin resistance (defined as the biologic response to a given concentration of the hormone is lower than expected) often occurs in patients with hypertension, which plays a key role in the pathogenesis of diabetes. ${ }^{17}$ Hypertension (15.4\%) had the highest prevalence rate among the 14 diseases investigated, and existed in a variety of comorbidity patterns, and the

Table 2 The Prevalence Rate of the Top 10 Binary Comorbidities Combinations

\begin{tabular}{|l|l|c|c|c|}
\hline Order & Comorbidity Mode & $\mathbf{N}$ & $\begin{array}{c}\text { Prevalence } \\
\text { Rate } \\
(\mathbf{n}=6754)\end{array}$ & $\begin{array}{c}\text { Accounts for the Proportion of Binary } \\
\text { Comorbidities Combinations (n=6 I6) }\end{array}$ \\
\hline 1 & Hypertension and dyslipidemia & 60 & $0.9 \%$ & $8.7 \%$ \\
\hline 2 & Hypertension and arthritis or rheumatism & 53 & $0.8 \%$ & $8.5 \%$ \\
\hline 3 & Stomach diseases and arthritis or rheumatism & 52 & $0.8 \%$ & $5.2 \%$ \\
\hline 4 & Hypertension and heart attack & 32 & $0.5 \%$ & $4.9 \%$ \\
\hline 5 & Hypertension and diabetes & 30 & $0.4 \%$ & $4.7 \%$ \\
\hline 6 & Hypertension and stoke & 29 & $0.4 \%$ & $3.4 \%$ \\
\hline 7 & Dyslipidemia and stomach diseases & 21 & $0.3 \%$ & $3.4 \%$ \\
\cline { 2 - 5 } & Dyslipidemia and diabetes & 21 & $0.3 \%$ & $3.1 \%$ \\
\hline 8 & Hypertension and stomach diseases & 19 & $0.3 \%$ & $2.9 \%$ \\
\hline 9 & Dyslipidemia and arthritis or rheumatism & 18 & $0.3 \%$ & $2.7 \%$ \\
\hline 10 & Dyslipidemia and heart attack & 17 & $0.3 \%$ & \\
\hline
\end{tabular}


Table 3 The Prevalence Rate of the Top 6 Ternary Comorbidities Combinations

\begin{tabular}{|c|c|c|c|c|}
\hline Order & Comorbidity Mode & $\mathbf{N}$ & $\begin{array}{c}\text { Prevalence } \\
\text { Rate }(n=6754)\end{array}$ & $\begin{array}{l}\text { Accounts for the Proportion of Ternary } \\
\text { Comorbidities Combinations }(n=275)\end{array}$ \\
\hline I & Hypertension and dyslipidemia and diabetes & 17 & $0.3 \%$ & $6.2 \%$ \\
\hline \multirow[t]{2}{*}{2} & $\begin{array}{l}\text { Hypertension and dyslipidemia and arthritis or } \\
\text { rheumatism }\end{array}$ & 13 & $0.2 \%$ & $4.7 \%$ \\
\hline & $\begin{array}{l}\text { Hypertension and heart attack and arthritis or } \\
\text { rheumatism }\end{array}$ & 13 & $0.2 \%$ & $4.7 \%$ \\
\hline 3 & $\begin{array}{l}\text { Hypertension and dyslipidemia and stomach } \\
\text { diseases }\end{array}$ & 12 & $0.2 \%$ & $4.4 \%$ \\
\hline 4 & Hypertension and dyslipidemia and stoke & 9 & $0.1 \%$ & $3.3 \%$ \\
\hline \multirow[t]{2}{*}{5} & $\begin{array}{l}\text { Hypertension and stomach diseases and arthritis or } \\
\text { rheumatism }\end{array}$ & 8 & $0.1 \%$ & $2.9 \%$ \\
\hline & Hypertension and dyslipidemia and heart attack & 8 & $0.1 \%$ & $2.9 \%$ \\
\hline \multirow[t]{5}{*}{6} & Hypertension and stomach diseases theart attack & 5 & $0.1 \%$ & $1.8 \%$ \\
\hline & $\begin{array}{l}\text { Hypertension and lung diseases and arthritis or } \\
\text { rheumatism }\end{array}$ & 5 & $0.1 \%$ & $1.8 \%$ \\
\hline & $\begin{array}{l}\text { Stomach diseases and lung diseases and } \\
\text { hypertension }\end{array}$ & 5 & $0.1 \%$ & $1.8 \%$ \\
\hline & $\begin{array}{l}\text { Stomach diseases and lung diseases and liver } \\
\text { diseases }\end{array}$ & 5 & $0.1 \%$ & $1.8 \%$ \\
\hline & Stomach diseases and dyslipidemia+diabetes & 5 & $0.1 \%$ & $1.8 \%$ \\
\hline
\end{tabular}

disease composition was complex. Blood pressure was an important risk factor for cardiovascular disease and mortality. ${ }^{18}$ Patients should enhance self-health management, consume less oil and salt in the diet, be active, make sure they get enough sleep and improve the quality of life. ${ }^{19}$ On the other hand, medical institutions should focus on such patients, early identification of high-risk groups to take measures to improve prognosis, community and health centers regularly organize education to do a good job of primary prevention. ${ }^{20}$ The standardized community management of hypertension can reduce the annual per capita drug treatment cost and annual per capita hospitalization cost by about $26 \mathrm{RMB}$ and $245 \mathrm{RMB}$, respectively, and save the annual per capita direct medical expenses of about $210 \mathrm{RMB}$, so that the output is greater than the input. ${ }^{21}$

As global life expectance rises, there was an increasing number of people suffering from chronic disorders, and so was China. When developing clinical guidelines to manage patients with comorbidity, consideration should be given to common conditions that lead to comorbidity and the ways in which these diseases are clustered. ${ }^{22}$ Compared with patients with a single disease, patients with comorbidity tended to face greater risks of poor health and healthcare needs, and disease prevention and management were more complex. ${ }^{7}$ In practice, improving healthcare utilization for patients with comorbidity is a challenging task. Issues such as polypharmacy pose enormous challenges for care coordination and patients' self-management, as most health systems are designed for managing single diseases. $^{23,24}$

The problem of comorbidity of middle-aged and elderly people in China has become more and more serious, but the current society still pays little attention to the prevention and control of chronic diseases. The general public has a weak self-protection awareness, and standardized clinical research on comorbidity has not been established. Adequate comorbidity information and good comorbidity management can improve the 
quality of life of this population ${ }^{25}$ and reduce medical costs. $^{26,27}$ Clinical decision-making for comorbid patients is complex and challenging, so understanding different profiles of comorbidity is essential for managing comorbidity more effectively and efficiently in health systems.

\section{Strengths and Limitations}

Understanding comorbidity patterns and underlying associated factors are of great importance for policy making purposes and clinical guidance to the rapidly growing aging population worldwide. To the best of our knowledge, this study was the latest to use a nationally data of middle-aged and elderly Chinese to evaluate the prevalence and common patterns of comorbidity. We described the different combinations of common diseases in detail to provide a reference for etiological research, combinatorial therapy and health management of comorbidities. CHARLS data have a large sample size and good representativeness, which can better reflect the overall prevalence of chronic diseases in China. Second, trained and qualified researchers collected all of the data by interactive face-to-face interviews to ensure that the information is correct.

There are also limitations to the study. Our study focused on the composition of the disease, so our analysis did not include socio-economic status of patients, detailed clinical information, and health-related behaviors, nor did it investigate potential confounding factors and consider the severity of disease. And recall bias may exist resulting from using self-reported methods to gather medical history.

\section{Conclusion}

Underpinned by a nationwide representative survey, we observed the prevalence of common chronic diseases in middle-aged and elderly people in China, and further explored the combination pattern of different diseases. The present study mainly revealed that: 1 ) The prevalence of 14 common chronic diseases was about $42 \%$; 2) About $40.0 \%$ of patients with chronic disease had comorbidity; 3 ) The most common binary comorbidities combinations was hypertension and dyslipidemia, and the most common ternary comorbidities combinations was hypertension and dyslipidemia and diabetes; 4) Hypertension exists in a variety of comorbidity patterns, and should be paid more attention to. Our findings underscore the importance of a shift from single disease-oriented clinical guidelines to a multiple-condition framework among middle-aged and elderly people in China.

\section{Ethical Approval}

CHARLS was approved by the Ethical Review Committee (IRB) at Peking University, Beijing, China. And we confirmed that the data accessed by the manuscript comply with relevant data protection and privacy regulations.

\section{Funding}

This research received no specific grant from any funding agency in the public, commercial, or not-for-profit sectors.

\section{Disclosure}

The authors report no conflicts of interest in this work.

\section{References}

1. Tang J, Liu WW. China's aging process and some misunderstandings on it. J Beijing Univ Technol. 2018;18:8-18.

2. National Bureau of Statistics. National data 2019. Available from: http://data.stats.gov.cn/easyquery.htm? $\mathrm{cn}=\mathrm{C} 01$. Accessed January 14, 2020.

3. World Health Organization. The World Health Report 2008. Primary Health Care (Now More Than Ever). Geneva: World Health Organization; 2008.

4. Sum G, Hone T, Atun R, et al. Multimorbidity and out-of-pocket expenditure on medicines: a systematic review. BMJ Global Health. 2018;3(1):e000505. doi:10.1136/bmjgh-2017-000505

5. Sum G, Salisbury C, Koh GC, et al. Implications of multimorbidity patterns on health care utilisation and quality of life in middle-income countries: cross-sectional analysis. J Glob Health. 2019;9(2):020413. doi:10.7189/jogh.09.020413

6. Yamada Y, Merz L, Kisvetrova H. Quality of life and comorbidity among older home care clients: role of positive attitudes toward aging. Qual Life Res. 2015;24(7):1661-1667. doi:10.1007/s11136014-0899-x

7. Barnett K, Mercer SW, Norbury M, et al. Epidemiology of multimorbidity and implications for health care, research, and medical education: a cross-sectional study. Lancet. 2012;380(9836):37-43. doi:10.1016/S0140-6736(12)60240-2

8. Disease and Injury Incidence and Prevalence Collaborators (Collaborators DaIIaP). Global, regional, and national incidence, prevalence, and years lived with disability for 354 diseases and injuries for 195 countries and territories, 1990-2017: a systematic analysis for the Global Burden of Disease Study 2017. Lancet. 2018;392(10159):1789-1858.

9. Tang C, Li MY, Wang B, et al. Prevalence of chronic disease among older people in Wuhan city and the knowledge, attitude, practice about chronic disease. Chin J Gerontol. 2017;37(5):1231-1234.

10. Schäfer I, von Leitner E, Schön G, et al. Multimorbidity patterns in the elderly: a new approach of disease clustering identifies complex interrelations between chronic conditions. PLoS One. 2010;5(12): e15941. doi:10.1371/journal.pone.0015941

11. Zhao Y, Hu Y, Smith JP, et al. Cohort profile: the China health and retirement longitudinal study (CHARLS). Int J Epidemiol. 2014;43 (1):61-68. doi:10.1093/ije/dys203

12. Hu X, Huang J, Lv Y, Li G, Peng X. Status of prevalence study on multimorbidity of chronic disease in China: systematic review. Geriatr Gerontol Int. 2015;15(1):1-10. doi:10.1111/ggi.12340 
13. Kim J, Keshavjee S, Atun R. Trends, patterns and health consequences of multimorbidity among South Korea adults: analysis of nationally representative survey data 2007-2016. J Glob Health. 2020;10(2):020426. doi:10.7189/jogh.10.020426

14. Kirchberger I, Meisinger C, Heier M, et al. Patterns of multimorbidity in the aged population. Results from the KORA-Age study. PLoS One. 2012;7(1):e30556. doi:10.1371/journal.pone.0030556

15. Wang R, Yan Z, Liang Y, et al. Prevalence and patterns of chronic disease pairs and multimorbidity among older Chinese adults living in a rural area. PLoS One. 2015;10(9):e0138521. doi:10.1371/journal. pone. 0138521

16. Sinnige J, Braspenning J, Schellevis F, Stirbu-Wagner I, Westert G, Korevaar J. The prevalence of disease clusters in older adults with multiple chronic diseases-a systematic literature review. PLoS One. 2013;8(11):e79641. doi:10.1371/journal.pone.0079641

17. Mancusi C, Izzo R, Di Gioia G, Losi MA, Barbato E, Morisco C. Insulin resistance the hinge between hypertension and type 2 diabetes. High Blood Press Cardiovasc Prev. 2020;27(6):515-526. doi:10.1007/s40292-020-00408-8

18. Oliveros E, Patel H, Kyung S, et al. Hypertension in older adults: assessment, management, and challenges. Clin Cardiol. 2020;43 (2):99-107. doi:10.1002/clc. 23303

19. Gu DF, Weng JP, Lu XF. Chinese guideline on healthy lifestyle to prevent cardiometabolic diseases. Chin Circ J. 2020;35(3):209-230.

20. Li X, Krumholz HM, Yip W, et al. Quality of primary health care in China: challenges and recommendations. Lancet. 2020;395 (10239):1802-1812. doi:10.1016/S0140-6736(20)30122-7
21. Liang X, Gu D, Zhang $\mathrm{H}$, et al. The analysis of drug cost and direct medical expense in community of health management of hypertensive patients. Chin J Prev Med. 2011;45(8):732-736.

22. Wang XW, Yao SS, Wang MY, et al. Multimorbidity among two million adults in China. Int $J$ Environ Res Public Health. 2020;17(10):3395. doi:10.3390/ijerph17103395

23. Pearson-Stuttard J, Ezzati M, Gregg EW. Multimorbidity-a defining challenge for health systems. Lancet Public Health. 2019;4(12): e599-e600. doi:10.1016/S2468-2667(19)30222-1

24. Tinetti ME, Fried TR, Boyd CM. Designing health care for the most common chronic condition-multimorbidity. JAMA. 2012;307 (23):2493-2494. doi:10.1001/jama.2012.5265

25. Bao X, Xie Y, Zhang X, et al. The association between multimorbidity and health-related quality of life: a cross-sectional survey among community middle-aged and elderly residents in southern China Health Qual Life Outcomes. 2019;17(1):107. doi:10.1186/s12955019-1175-0

26. Zhao Y, Atun R, Oldenburg B, et al. Physical multimorbidity, health service use, and catastrophic health expenditure by socioeconomic groups in China: an analysis of population-based panel data. Lancet Global Health. 2020;8(6):e840-e849. doi:10.1016/S2214-109X(20) 30127-3

27. Wu XL, Xu H, Yu Q, et al. Evaluation of intervention effect of health management on chronic diseases in the elderly. Chin J Dis Control Prev. 2018;22(6):573-576.
International Journal of General Medicine

\section{Publish your work in this journal}

The International Journal of General Medicine is an international, peer-reviewed open-access journal that focuses on general and internal medicine, pathogenesis, epidemiology, diagnosis, monitoring and treatment protocols. The journal is characterized by the rapid reporting of reviews, original research and clinical studies

\section{Dovepress}

across all disease areas. The manuscript management system is completely online and includes a very quick and fair peer-review system, which is all easy to use. Visit http://www.dovepress.com/ testimonials.php to read real quotes from published authors. 